\title{
SYMMETRY PRESERVING IN TOPOLOGICAL MAPPING FOR TENSION \\ STRUCTURES
}

Juan F. Carbonell-Márquez ${ }^{1}$, Rafael Jurado-Piña ${ }^{2}$, Luisa. M. Gil-Martín ${ }^{3}$, and Enrique Hernández-Montes ${ }^{4}$

(Published in “Engineering Structures”, Volume 52, July 2013, Pages 64-68)

http://dx.doi.org/10.1016/j.engstruct.2013.02.011

\section{Keywords}

Tension structures; form-finding; symmetry; Topological Mapping; force density method;

\begin{abstract}
When using Topological Mapping (TM) together with the Force Density Method (FDM) in order to find a first form of equilibrium for a tension structure whose fixed nodes coincide in plan view with the vertices of a regular polygon, it has been observed that the symmetry may be lost. The problem is related to the mapping procedure that is introduced in the process. However, if some rules are followed in constructing the topology for the mesh, the symmetry can be preserved. With a symmetric mesh, the later cutting pattern generation process becomes
\end{abstract}

\footnotetext{
${ }^{1} \mathrm{PhD}$ Candidate, Department of Structural Mechanics, University of Granada (UGR). Campus Universitario de Fuentenueva s/n. 18072 Granada, Spain. (corresponding author). Email jfcarbonell@ugr.es. Tel.: +34 958249965; fax: +34 958249959
}

${ }^{2}$ Associate Professor, Department of Civil Engineering-Transport. Technical University of Madrid (UPM), C/ Profesor Aranguren, s/n, 28040 Madrid, Spain rjurado@caminos.upm.es

${ }^{3}$ Associate Professor, Department of Structural Mechanics, University of Granada (UGR). Campus Universitario de Fuentenueva s/n. 18072 Granada, Spain. mlgil@ugr.es.

\footnotetext{
${ }^{4}$ Professor, Department of Structural Mechanics, University of Granada (UGR). Campus Universitario de Fuentenueva s/n.18072 Granada, Spain. emontes@ugr.es.
} 
simpler, easier, and cheaper. This paper will provide one simple algorithm that has been proved to work effectively in preserving the symmetry in the cited type of tension structures. Several examples are presented in order to better illustrate the applicability of these easy prescriptions.

\section{Introduction: Topological Mapping for tension structures}

The use of Topological Mapping (TM) for tension structures has been introduced [1] as a mapping procedure for the Force Density Method (FDM) [2][3]. TM is applicable only to FDM as a means of obtaining a first equilibrium shape employing topological rules, i.e. to do the form-finding process. As seen through the many examples presented in our literature, for instance, in [4], an initial guess of the shape is needed in order to generate a network. The advantage of TM-FDM is that an initial guess is not needed; only the connectivity of the nodes is necessary to find the solution to the problem in contrast to the mapping methods that are based exclusively on geometry. The only inputs to trigger the TM-FDM are the coordinates of the fixed nodes, the force-length or force density ratios for the net branches and a series of topological rules that define a matrix which contains the information for the connectivity of the net nodes. Once the geometry and the forces in the branches are determined by means of FDM, the cable model can be transformed into a superficial finite elements model using the planar triangular finite element with constant stresses and strains. This conversion is explained in further detail in [5]. For more formulations, examples, and details of FDM, the reader may consult references [1-3] and [6-9].

TM provides the connectivity of the network according to a set of given data: the number of nodes in the first step, the number of steps, the type of relationship between consecutive steps and the alternative to close or open network.

Three types of relationships are defined between steps: A, B and C. Relationship A corresponds to a pattern in which each node at a given step is connected to three more nodes of the following step. In the case of relationship B, each node at a given step is connected to two nodes of the following step. Case C is such that each node at a given step is connected alternatively to one or 
to three nodes of the next step [1][7]. For the three types A, B, and C, each node is connected to the adjacent ones on the same step. Fig. 1 (a) and (b) shows the typical relationships or basic networks A and B.

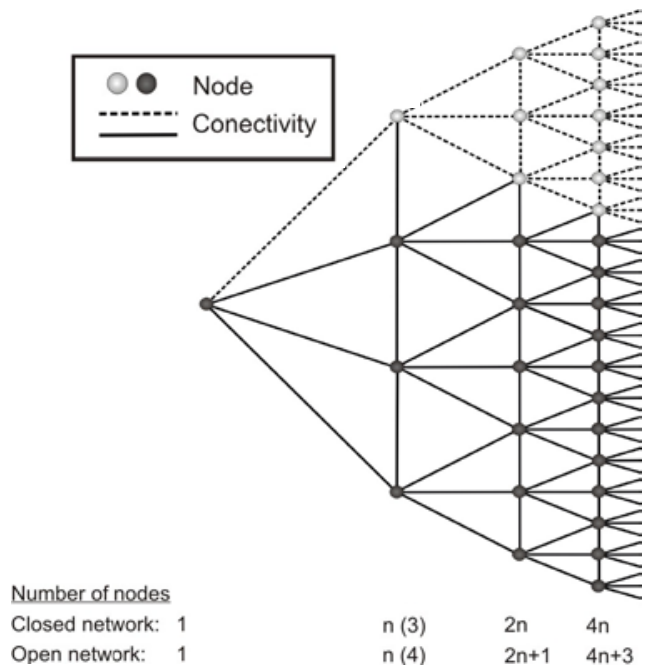

(a)

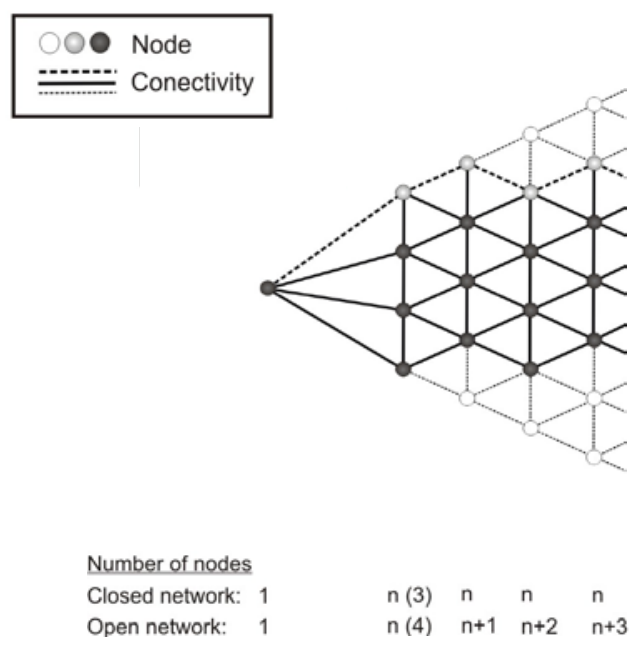

(b)

Fig.1. Types of basic networks: (a) Type A; (b) Type B. From [1]

As described earlier, two possible types of networks are possible: open and closed. These two types of networks are related to the contour of the equilibrium shape [1]. First, the closed networks are those for which the nodes located in the last step constitute the contour of the equilibrium shape. The contour of the equilibrium shape for an open network is formed by the nodes of the contour of the topology network (see Fig. 2). Different colours of nodes in Fig. 1 (a) and (b) depend on the type of network employed. The present study deals only with the closed networks of the A and B relationship. Therefore, steps will be referred to as rings hereafter. For further details on TM, the reader should consult references [1] and [7].

Different basic networks may be used together in order to get a combined topology. In this case, it is necessary to define the desired sequence of combinations (for example A-A-B-B-B). This sequence will give the relationships between nodes of consecutive rings. Therefore, if the mesh has $N$ rings, the sequence of topology consists in $N-1$ letters indicating A or B relationships. 


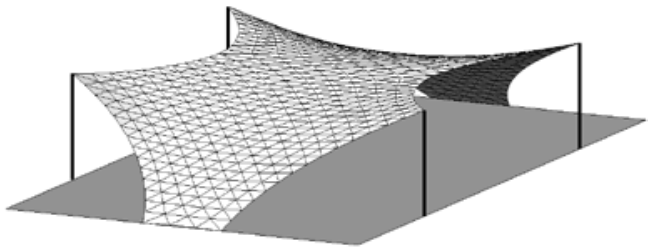

(a)

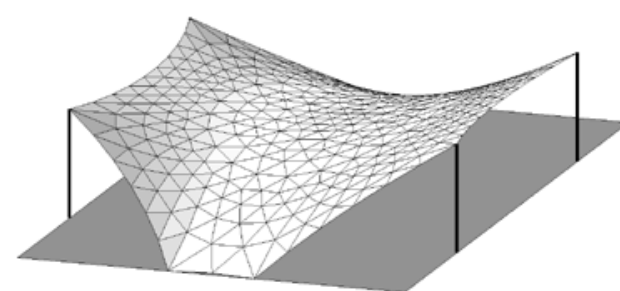

(b)

Fig. 2. Types of network for the same structure: (a) open network; (b) closed network. From [1]

It is important to point out that, the fixed points are assigned to the nodes of the network through the calculation of the distance between consecutive fixed points and the computation of the perimeter formed by them so that the distribution of nodes of the last ring is done proportionally to the real distance between the fixed points.

According to the above mentioned, the process for generating closed networks requires a reduced and simple data input: the location of the fixed nodes, the force densities of the interior and exterior branches and the topology of the net given by the sequence of combinations between rings.

\section{The loss of symmetry}

Let us imagine that we are dealing with a tension structure in which the floor plan is a regular polygon. Once the equilibrium shape of the tension structure has been found with TM-FDM, the structural shape has to be converted into a set of planar cloths for its fabrication [7]. This is a process called cutting pattern generation [10][11]. As this is not a simple process, one may take advantage of the symmetry of the tension structure since a non-symmetric mesh may generate different patterns leading to a more expensive process.

However, it is observed that depending on the adopted sequence of relationships between rings, the mesh may or may not be symmetric. Fig. 3 shows two meshes with symmetric supports; the only difference between them is the sequence of relationships between rings: B-A-A for Fig. 3 (a) and A-A-B for Fig. 3 (b). As a consequence, Fig. 3 (a) is symmetric whereas Fig. 3 (b) is not. 


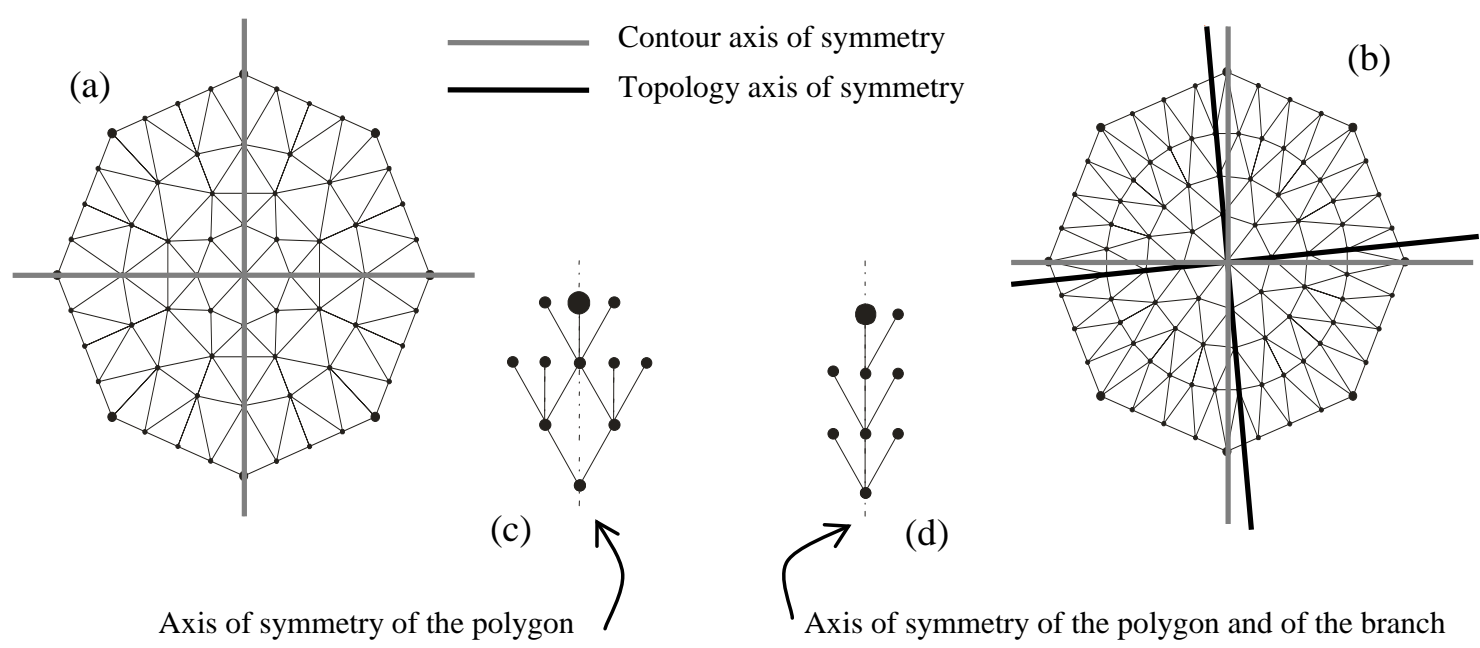

Fig. 3. Two different topologies for the same structure: (a) \& (c) mesh and branch for B-A-A; (b) \& (d)

A-A-B

By observing how the mesh of Fig. 3 (b) is built ring by ring (Fig. 4 (a)), it can be noticed that this is always a symmetric procedure. According to this, the topology of the mesh will always be symmetric. However, when the last ring is reached (see Fig. 4 (b)), their nodes must take up their prescribed distribution according to the space between fixed nodes, as explained earlier, making the mesh asymmetric. Therefore, the key point to preserving the symmetry of the topology is the spatial symmetric distribution which needs to be compatible with the prescribed distribution of the contour of the tension structure. This compatibility will be reached if the axis of symmetry of the mesh and the regular polygon of the contour coincide after distributing and positioning the mesh nodes -including the contour nodes- (Fig. 3).
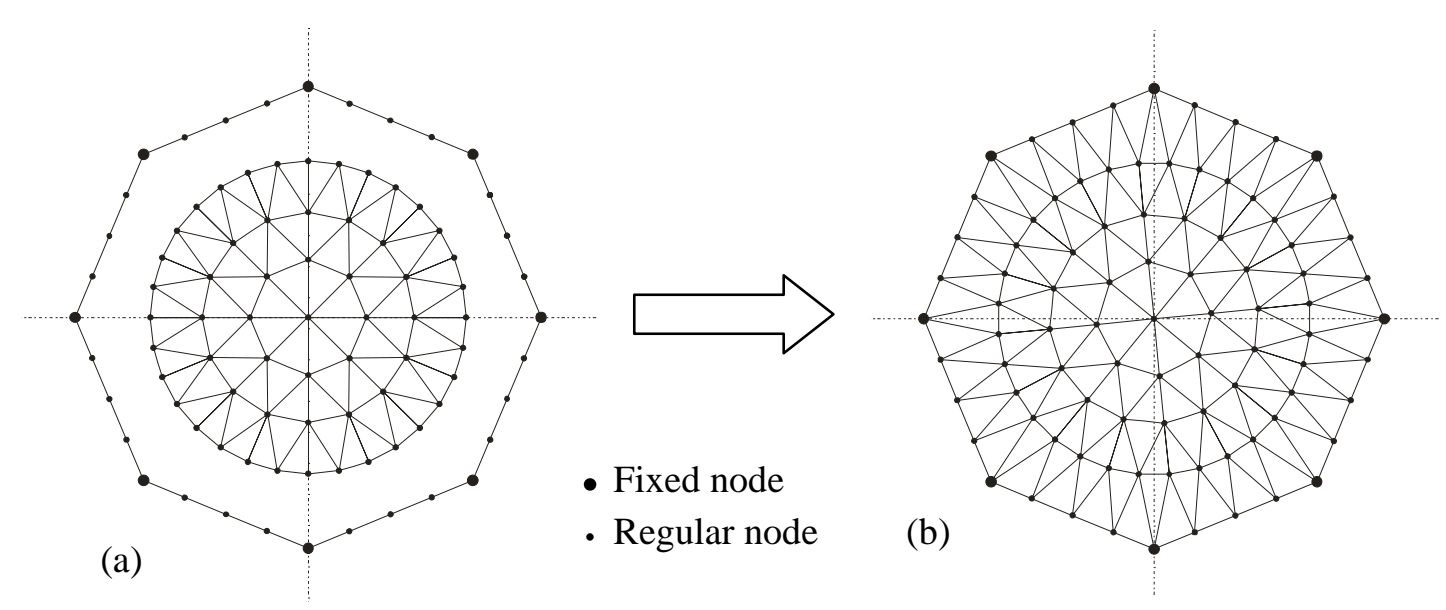
Fig. 4. Building of a mesh: (a) Network sketch without connections between penultimate and last rings; (b) Complete network sketch with symmetric internal distribution but last connections with an asymmetric layout

\section{A proposed algorithm for preserving symmetry}

Once the reasons for loss of symmetry in the mesh construction have been explained, a new algorithm for preserving the symmetry is presented. This algorithm will let the designer know if the topology introduced in the FDM produces as a result a symmetric structure without needing to visualize the entire mesh.

Fig. 1 shows how relationships A and B work. Starting from the first ring nodes, the mesh grows outwards. Thereby, the mesh may be understood as a core linked to the exterior fixed nodes by means of a number of branches. These branches will be joined together so that all the nodes of the contour remain linked to the core. Fig. 5(a) shows the case for a pentagonal-shaped structure. Here there are five rings with a sequence B-B-A-A. The branches that link the exterior points located in the axis of symmetry are illustrated by thick lines. These branches can be plotted separately from the remainder of the mesh by knowing the sequence that defines the relationship between rings, see Fig. 5(b). In doing so, if one of the fixed nodes of the contour belonging to the axis of symmetry is reached in a symmetric way, without altering the symmetry, the entire mesh will be symmetric.

In order to visualize some examples of the construction of the referred branch plot, the corresponding branches are also represented for the two different meshes given in Fig. 3 (a) and (b). The plot corresponding to mesh (a) is (c) and the one corresponding to (b) is plot (d). 


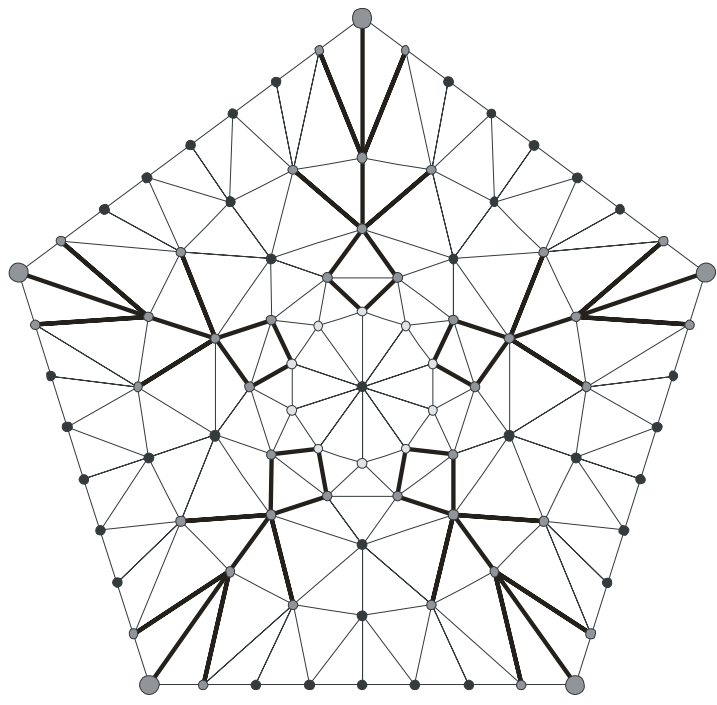

(a)
- Core node

- Branch node

Branch link

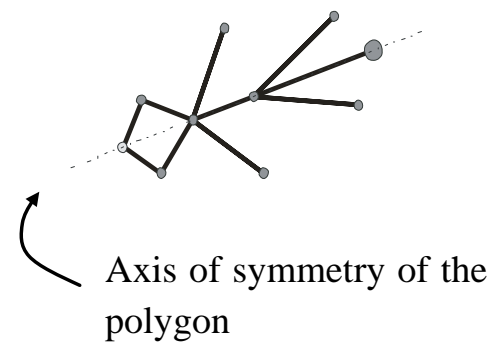

(b)

Fig. 5. (a) Topology for a pentagonal-shaped structure; (b) One of the branches

It must be pointed out that the number of nodes in the first ring cannot be any number, but rather it must be the same or a multiple of the contour number of sides. Nevertheless, this rule alone does not guarantee symmetry.

From the above observations, the algorithm has already been established with the procedure presented in the flow charts in Fig. 6. The algorithm is presented in two different ways: (a) graphically, as originally conceived, and (b) analytically so that it can be easily implemented in any computer language for its programming. The first way consists of plotting a singular branch of the mesh according to some given prescriptions. If the last ring is reached with a node in the axis with the plot of the branch being symmetric, as stated above, the network will be symmetric. On the other hand, the second method starts with the storage of the topology sequence in a vector called SeqVec. Later on, an iterative process controlled by the counter it, will fill a vector with the same number of components as the number of rings, NumRing. Each of these components is related to each ring and they will be equal to 1 or 2 depending on the type (A or B) of the former one. If a 1 is assigned to last ring, the entire mesh results symmetric. 


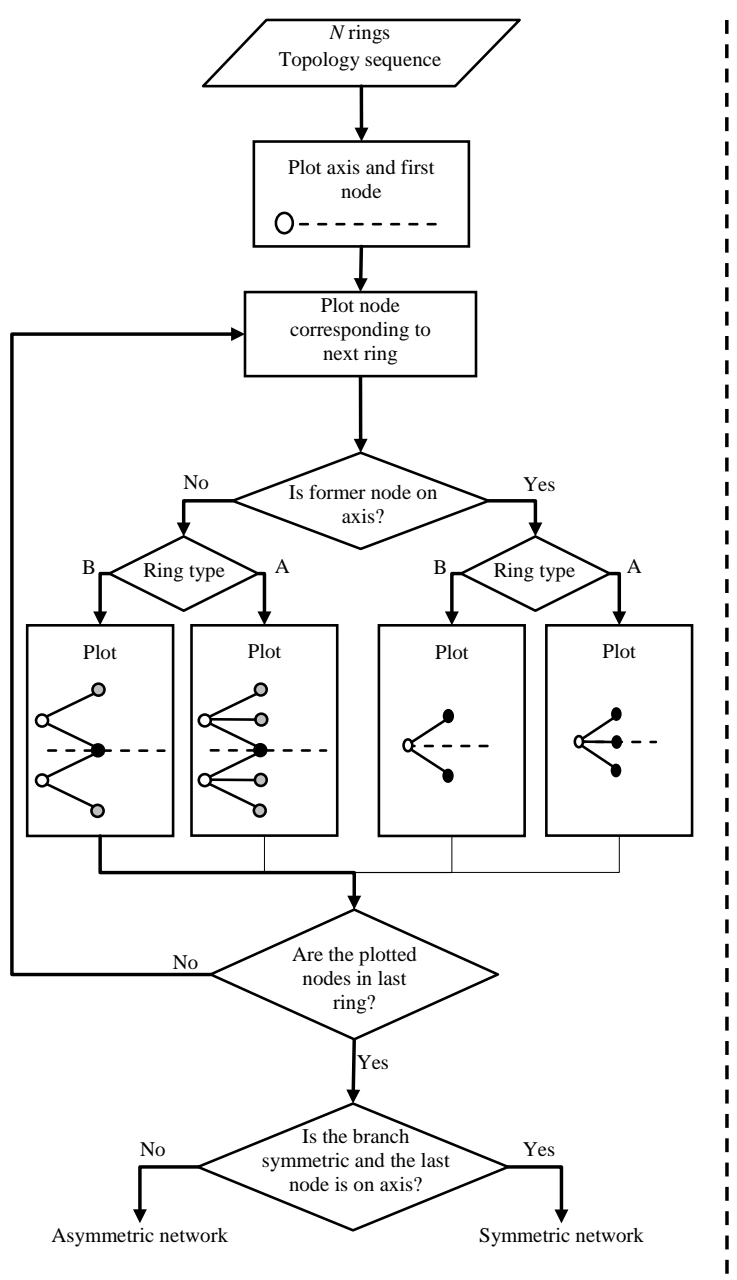

(a)

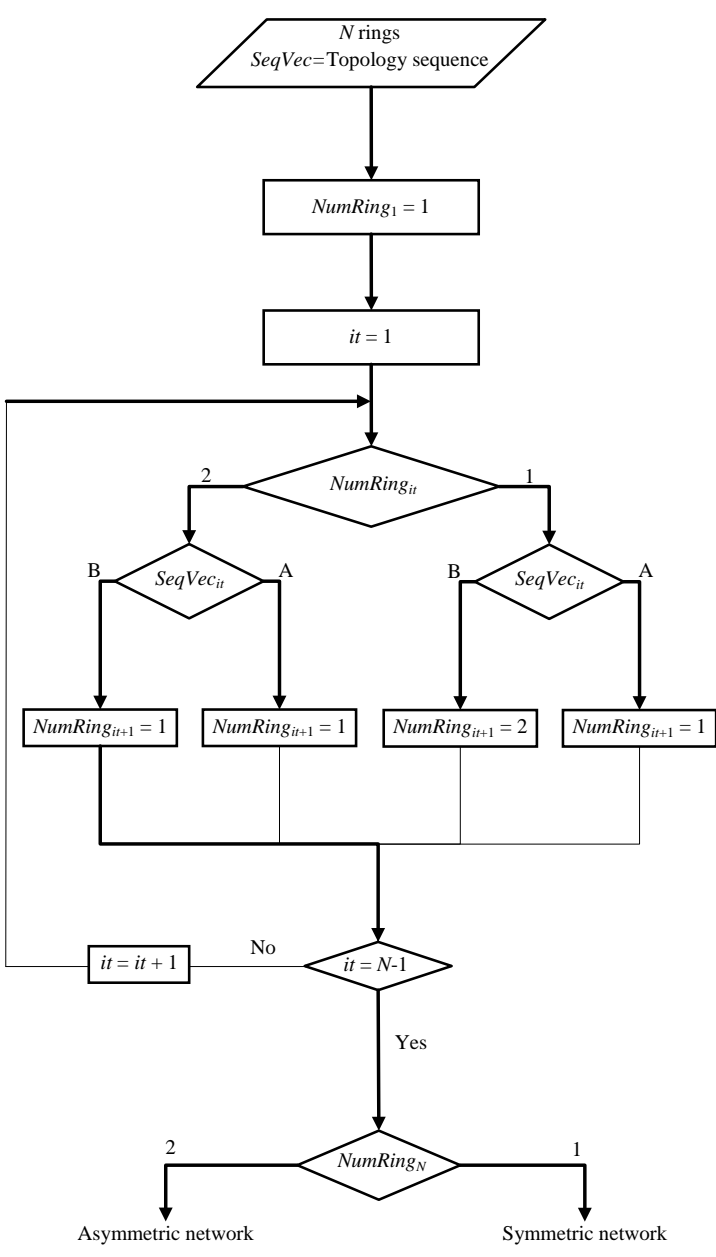

(b)

Fig. 6. Proposed algorithm for analyzing the network to be used: (a) graphically; (b) analytically

Although the approach is only valid in tension structures whose fixed nodes coincide with the vertices of a regular polygon, these kinds of tension structures are commonly used and, therefore, the field of application of the method is still important.

\section{Example}

It has been explained above that the preservation of symmetry is a basic element for making the pattern generation task easier.

Fig. 7 shows an octagonal-shaped tension structure that has been materialized by means of 13 rings with 8 nodes in the first ring. The relationship between the rings is B-A-B-B-A-B-A-B-BA-B-B. As one may appreciate in figure (Fig. 7 (a)), the mesh is symmetric. However there is 
no need to plot the entire network since the symmetry may be evaluated with the presented algorithm (Fig. 7 (d)).

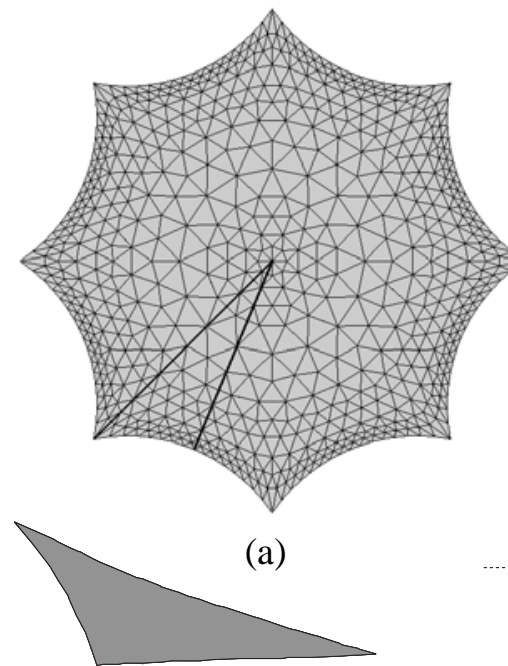

(c)

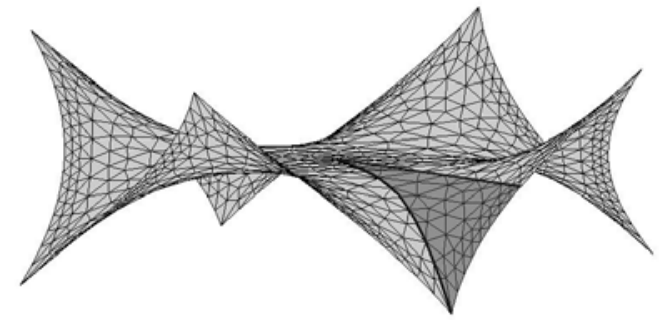

(b)

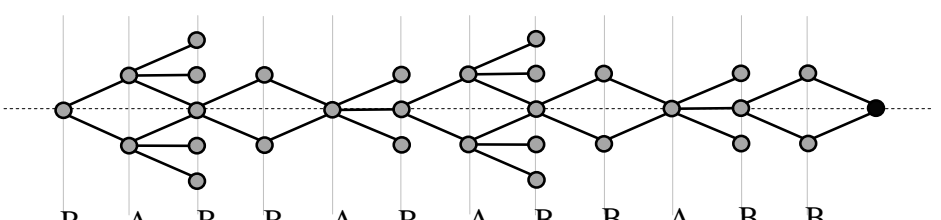

$\begin{array}{llllllllllll}\text { B } & \text { A } & \text { B } & \text { B } & \text { A } & \text { B } & \text { A } & \text { B } & \text { B } & \text { A } & \text { B } & \text { B }\end{array}$

(d)

Fig. 7. Symmetric tension structure: (a) plan view; (b) perspective; (c) flattened pattern; (d) branch plot

Fig. 7 (c) also shows the only pattern needed for the construction of the whole tension structure. Using this pattern 16 times, the tension structure will materialize.

\section{Conclusions}

Preserving the symmetry in tension structures is extremely useful; it makes the patterning simpler, easier, and cheaper since it allows building the tension structure with just a few different patterns.

Logically, tension structures whose fixed nodes (or its projections) are the vertices of a regular polygon should be symmetric. However, when applying Topological Mapping (TM) and the Force Density Method (FDM) for form-finding it has been observed that in many cases the resulting tension structures become asymmetric. Preserving symmetry is therefore of great interest in the mapping of tension structures.

The reasons for this loss of symmetry have been explained and a new algorithm for preserving the symmetry has been presented. The rules for the algorithm are related to the sequence for the 
relationship between the rings and the number of nodes of the first ring. A simple and useful rule is given in order to let the user know if the topology to be introduced in FDM is symmetric.

\section{Acknowledgements}

This research work was carried out under the financial support provided by Spanish Ministry of Education and Science as part of the Research Projects BIA 2007-62595. The first author is a Spanish Government PhD fellow (FPU grant AP 2010-3707). Their support is gratefully acknowledged.

\section{References}

[1] Hernández-Montes, E, Jurado-Piña, R, and Bayo, E. Topological mapping for tension structures. Journal of Structural Engineering 2006; 132(6): 970-977.

[2] Linkwitz, K, and Schek, HJ. Einige Bemerkung von vorsgepannten Seilnetzkonstruktionen. Ingenieur-archv 1971; 40: 145-158.

[3] Schek, HJ. The force density method for form-finding and computation of general networks. Computer Methods in Applied Mechanics and Engineering 1974; 3: 115-134.

[4] Levy, R, and Spillers, WR. Analysis of geometrically nonlinear structures, $2^{\text {nd }}$ Ed. London: Chapman \& Hall; 2004.

[5] Lai, C. Y., You, Z., Pellegrino, S. Shape and stress analysis of symmetric CRTS reflectors (CUED/D-STRUCT/TR170). Cambridge: European Space Agency Contractor Report; 2001

[6] Linkwitz, K. About formfinding of double-curved structures. Engineering Structures 1999; 21(8): 709-718.

[7] Jurado-Piña, R, Hernández-Montes, E, and Gil-Martín, LM. Topological mesh for shell structures. Applied Mathematical Modelling 2009; 33(2): 948-958.

[8] Koohestani, K. Form-finding of tensegrity structures via genetic algorithm. International Journal of Solids and Structures 2012; 49: 739-747. 
[9] Sánchez, J, Serna, MÁ, Morer, P. A multi-step force-density method and surface-fitting approach for the preliminary shape design of tensile structures. Engineering Structures 2007; 29 (8): 1966-1976.

[10] Moncrieff, E, and Topping, BHV. Computer methods for the generation of membrane cutting patterns. Computers \& Structures 1990; 37(4): 441-450.

[11] Kim, J-Y, Lee, J-B. A new technique for optimum cutting pattern generation of membrane structures. Engineering Structures 2002; 24(6): 745-756. 